\title{
Motional macroscopic quantum superposition states of a trapped three-level ion
}

\author{
Ö. E. Müstecaplı̆ğlu \\ Koç University, Rumelifeneri Yolu, 34450 Sarlyer, Istanbul, Turkey \\ (Received 28 February 2003; revised manuscript received 6 May 2003; published 27 August 2003)
}

\begin{abstract}
We investigate quantum dynamical properties of a trapped three-level ion interacting with two laser beams in $\Lambda$ configuration. A unitary transformation method is developed to study the interaction of the ion with its vibrational phonons, quanta of ion's own center-of-mass motion. Under certain conditions on laser parameters, this interaction is shown to be unitarily equivalent to two-phonon cascade transitions. Complicated temporal behaviors of level populations and mean number of phonons are described clearly by identifying dynamical variables of the cascade model as building blocks. Furthermore, analyzing quantum states of vibrational phonons by Husimi- $Q$ function, we find that at times, determined by the underlying cascade dynamics, twoand three-component macroscopic quantum superposition states can be obtained depending on the Lamb-Dicke parameter and the initial conditions of the system. A wide range of initial conditions and experimental parameters are discussed using both exact and analytical solutions. Alternative routes to reach the target states are found.
\end{abstract}

DOI: 10.1103/PhysRevA.68.023811

\section{INTRODUCTION}

Advances in ion trapping and laser cooling technology [1-3] allows for the realization of strictly nonclassical states, such as entangled [4], Fock number [5], spin-oscillator [6] and even-odd coherent states [7], of motion of trapped cold ions. Local character of trapped ion leads to the quantization of its center-of-mass motion in terms of vibrational phonons. Extremely weak damping and decoherence experienced by the vibrational phonons [8] make it easier to prepare and detect such delicate profoundly quantum states in ion traps in comparison to other systems such as optical cavities or solidstate heterostructures. Which system or combination of various systems would be most suitable for technological applications is a question of practical importance. Ion trap systems can be utilized to improve our understanding of nonclassical states and quantum decoherence. It is suggested that one can deliberately induce damping into the ion trap system and study engineered decoherence using Schrödinger's catlike states [7], which are superpositions of macroscopically distinct coherent states [9]. Besides their fundamental importance, cat states can be used in testing quantum measurement theories, in applications of matter interferometers [10], and for quantum computation [12]. Various proposals to construct two-component cat states in trapped two-level ion systems have been discussed extensively in the last decade [10-19].

In schemes of nonclassical state generation in ion traps, external classical driving fields are used to manipulate coupling internal electronic levels of the ion with its vibrational phonons. Physical models of such systems contain exponential nonlinearities in the motional degrees of freedom. For linearization, one may consider the strong excitation regime or the Lamb-Dicke limit (LDL). In the strong excitation regime, driving field intensities are so high that the Rabi frequency of ion-laser interaction becomes much larger than the ion trap frequency. LDL is established when laser wavelength is much larger than ion confinement. Quantum properties of ion motion are most prominent in weak excitation regime. It was recently shown that there exists a unitary dy-
PACS number(s): 42.50.Ct, 32.80.Pj, 42.50.Vk

namical equivalence between Jaynes-Cummings model [20] of cavity quantum electrodynamics (QED) and the trapped two-level ion system [21] in the weak excitation regime. This is used to predict superrevivals of the mean number of vibrational phonons, which is originally known to occur in cavity photons with sub-Poissonian distributions [22]. Subsequently, the unitary transformation technique has been widely used to explore other time-varying properties of trapped two-level ions [23]. Generalizations to trapped twolevel $N$-ion system [24] and trapped neutral atoms [25] were developed.

In this paper, we present a unitary transformation method to investigate dynamical properties of a trapped three-level ion optically driven in $\Lambda$ configuration. We will show that both two- and three-component macroscopic quantum superposition (Schrödinger cat) states of the vibrational phonons can be generated with the same initial state depending on the value of the Lamb-Dicke parameter. We shall further demonstrate that the time at which cat states appear can be determined by the unitary dynamical equivalent of the system, which turns out to be the two-phonon cascade in our case. Such multiple-component generalizations of the catlike states have been considered before in the context of cavity QED systems [26] and trapped ion systems [27]. In the cavity QED scheme [26], a cat state is created in the cavity field using multiple three-level atoms passing through a cavity at equal interaction times. Three-level atoms are reduced to two-level atoms under an adiabatic elimination. In the trapped ion proposal, linear superpositions of coherent states in the phase space are discussed [27]. Both proposals are based upon sequential steps of laser operations. The experimental demonstration [7] of two-component cat states is also based upon a sequential procedure. Increase of the number of laser operations and steps in such procedures limit the generalizations to higher excitations, shorter time operation, and larger macroscopic states that could allow direct detection. Present paper differs from the above proposals and the experiment as it is based upon deterministic creation of cat states in a single step. In the experiment, additional laser operations are needed to simulate the effect of displacement 


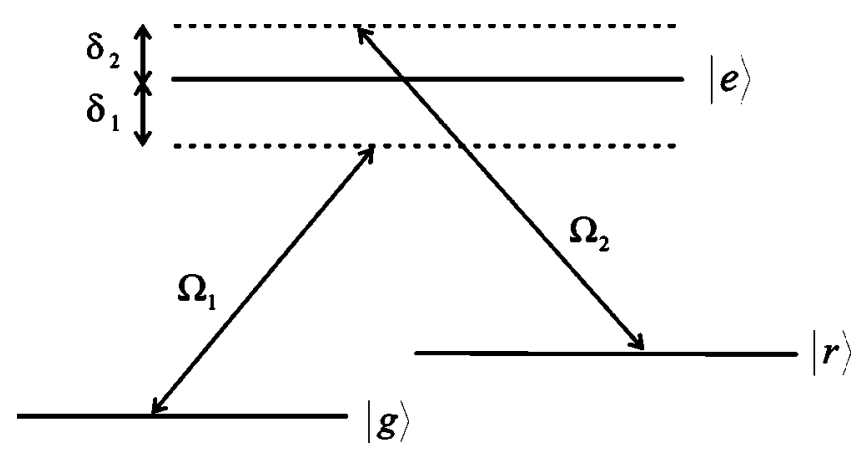

FIG. 1. Three-level ion interacting with two laser pulses in a $\Lambda$ configuration.

operators by tuning the Raman pulses appropriately. In the unitary transformation method, such preparation and displacement steps are contained within the transformation matrix while the transformed cascade model generates the desired dynamics. This is how present method reduces the number of laser operations yet maintaining a similar stepwise strategy.

Other differences between the present paper and the proposals on two- and multiple-component cat states as well as the experimentally used one can be summarized as follows. Coherent states in our study are superposed on a ring in the phase space, which is suitable for number squeezing and amplitude squeezing applications, and not possible with linearly superposed states. We demonstrate tunable generation of two- and three-component states at a single ion with the same initial state depending on easily controllable system parameters. We also note the alternative routes which can provide flexibility in experiments. The present method does not rely on standard approximations such as adiabatic elimination or rotating wave approximation (RWA) and is suitable for both small and large number of vibrational phonons in coherent states.

Finally, let us emphasize that in addition to the cat state results, the paper develops a systematic engineering of threelevel Hamiltonians. Engineering two-level Hamiltonians has been considered by approximate schemes before [13] and recently by unitary transformation methods [23]. Present paper eliminates the need for standard adiabatic elimination approximation and goes beyond the LDL. Full three-level ion dynamics with and without rotating wave approximation has been characterized.

The paper is organized as follows. In Sec. II the physical model is introduced and unitary transformation method is developed. Numerical results and their discussions are given in Sec. III. Finally, we summarize the results and conclude in Sec. IV.

\section{PHYSICAL MODEL AND THEORY}

We consider an ion trapped in a harmonic potential and driven by two classical laser fields as depicted in Fig. 1. We assume that harmonic potential frequencies are designed to construct a linear trap so that the center-of-mass (c.m.) motion of the ion is effectively one dimensional along the trap axis ( $x$ axis). Neglecting tightly confined transverse motion, the system is described by the Hamiltonian in units where $\hbar=1$,

$$
H=H_{C M}+H_{i o n}+H_{1}+H_{2},
$$

where

$$
\begin{gathered}
H_{C M}=\frac{p_{x}^{2}}{2 m}+\frac{1}{2} m \nu^{2} x^{2} \\
H_{i o n}=\omega_{e g} R_{e e}+\left(\omega_{e g}-\omega_{e r}\right) R_{r r} \\
H_{1}=\frac{\Omega_{1}}{2} e^{i\left( \pm k_{1} x-\omega_{1} t\right)} R_{e g}+\text { H.c. } \\
H_{2}=\frac{\Omega_{2}}{2} e^{i\left( \pm k_{2} x-\omega_{2} t\right)} R_{e r}+\text { H.c. }
\end{gathered}
$$

We introduced $R_{a b}=|a\rangle\langle b|$ as the ionic transition operators for the levels $a, b=g, r, e$, with $R_{g g}+R_{r r}+R_{e e}=1$. The ionic internal level energies $\omega_{a g}$ are measured from the ground level. Two laser beams $(i=1,2)$ are characterized by their wave numbers $k_{i}>0$ and frequencies $\omega_{i}$. Rabi frequencies of the dipole interactions between the lasers and the ion are given by $\Omega_{i}$. It is implicitly supposed that harmonic trap frequency $\nu$ is the same for all levels. Level dependence of trap frequencies can be more significant in the case of neutral atoms where this may induce pairwise correlations among the vibrational phonons [25].

Quantum-mechanical investigation of the ionic c.m. motion can be performed by the standard harmonic-oscillator quantization of $H_{C M}$ via $x=\sqrt{1 / 2 m \nu}\left(a+a^{\dagger}\right)$ and $p_{x}$ $=i \sqrt{m \nu / 2}\left(a^{\dagger}-a\right)$. Bosonic operators $a, a^{\dagger}$, which obey the usual Weyl-Heisenberg algebra, are the annihilation and creation operators of the vibrational phonons, respectively. Introducing the Lamb-Dicke parameters $\eta_{i}=k_{i} \sqrt{1 / 2 m \nu}$, the Hamiltonian of the system can be written as

$$
H=H_{0}+V
$$

where

$$
\begin{gathered}
H_{0}=\nu a^{\dagger} a-\delta_{1} R_{e e}-\left(\delta_{1}-\delta_{2}\right) R_{r r}, \\
V=\frac{\Omega_{1}}{2} e^{ \pm i \eta_{1}\left(a^{\dagger}+a\right)} R_{e g}+\frac{\Omega_{2}}{2} e^{ \pm i \eta_{2}\left(a^{\dagger}+a\right)} R_{e r}+\text { H.c. }
\end{gathered}
$$

We applied a rotating frame transformation by $U_{0}$ $=\exp \left[-i \omega_{1} t R_{e e}-i\left(\omega_{1}-\omega_{2}\right) R_{r r}\right]$. Here the detunings are $\delta_{1}$ $=\omega_{1}-\omega_{e g}, \delta_{2}=\omega_{2}-\omega_{e r}$. We now remove the Bose variables from the interaction part of the Hamiltonian. A unitary transformation method to accomplish this has been developed originally for the case of a two-level ion [21] and generalized to neutral two-level atoms [25]. The success of the method lies in the fact that bosonic (external/c.m.) degrees of freedom enter into the interaction $V$ in the form of Glauber displacement operators $D(\alpha)=\exp \left(\alpha a^{\dagger}-\alpha^{*} a\right)$ with $\alpha=$ $\pm i \eta$. This permits the construction of a unitary matrix involving displacement operators with purely imaginary parameters $\beta$ so that due to the property $D(\alpha) D(\beta)=D(\alpha$ 
$+\beta$ ), we can remove all the exponential terms in $V$. It should be noted that external degrees of freedom are not eliminated from the whole system, as it is usually done in the standard approximate (Markovian or semiclassical) approaches in quantum optics [28]. While they are entirely removed from $V$, new contributions appear in $H_{0}$ because of the transformation of the free field energy $\nu a^{\dagger} a$. They arise due to the property $D(\alpha) a^{\dagger} a D(\alpha)=a^{\dagger} a+\alpha\left(a^{\dagger}-a\right)-\alpha^{2}$ where the second and the last terms are, respectively, the recoil momentum and Doppler shifts in the free field energy due to the ionic motion. Despite these additional terms, the unitary transformation method is beneficial due to trading off the exponential complexity in $V$ with a polynomial one in $H_{0}$, while still being exact.

Following this strategy, we find that there exists a general class of transformations $U_{E}$ which diagonalize $V$ identically such that having $\widetilde{V}=U_{E}^{\dagger} V U_{E}$, we get

$$
\widetilde{V}=\frac{\Omega}{2}\left(R_{r r}-R_{g g}\right) .
$$

Here $\widetilde{V}$ consists of only ionic internal degrees of freedom with $\Omega=\sqrt{\left|\Omega_{1}\right|^{2}+\left|\Omega_{2}\right|^{2}}$. The unitary transformation matrix can be expressed as a product of two matrices $U_{E}=T E$ given by

$$
\begin{gathered}
T=\frac{\Omega_{2}^{*}}{\sqrt{2} \Omega} D\left(-i \eta_{2}\right)\left(R_{r r}-R_{r g}\right) \\
+\frac{\Omega_{2}}{\Omega} R_{g e}+\frac{\Omega_{1}^{*}}{\sqrt{2} \Omega} D\left(-i \eta_{1}\right)\left(R_{g r}-R_{g g}\right)+\frac{1}{2}\left(R_{e r}+R_{e g}\right) \\
-\frac{\Omega_{1}}{\Omega} D\left[i\left(\eta_{1}-\eta_{2}\right)\right] R_{r e} \\
E=E_{g} R_{g g}+E_{r} R_{r r}+E_{e} R_{e e} .
\end{gathered}
$$

Here, $E_{j}=\exp \left(i \theta_{j}\right)$ and $\theta_{j}$ are arbitrary unitary and Hermitian operators, respectively, to be specified later. We can exploit their arbitrariness, in order to convert the transformed $H_{0}$ into an appropriate simple model Hamiltonian. Let us first consider the off-diagonal elements in $\widetilde{H}_{0}=U_{E}^{\dagger} H_{0} U_{E}$,

$$
\begin{aligned}
\left(\widetilde{H}_{0}\right)_{e r}= & -\frac{\Omega_{1}^{*} \Omega_{2}^{*}}{\sqrt{2} \Omega^{2}}\left[E_{e}^{\dagger} D^{\dagger}\left(i \eta_{12}\right)(\nu n-\Delta) D\left(-i \eta_{2}\right) E_{r}\right. \\
& \left.-\nu E_{e}^{\dagger} n D\left(-i \eta_{1}\right) E_{r}\right], \\
\left(\widetilde{H}_{0}\right)_{e g}= & \frac{\Omega_{1}^{*} \Omega_{2}^{*}}{\sqrt{2} \Omega^{2}}\left[E_{e}^{\dagger} D^{\dagger}\left(i \eta_{12}\right)(\nu n-\Delta) D\left(-i \eta_{2}\right) E_{g}\right. \\
& \left.-\nu E_{e}^{\dagger} n D\left(-i \eta_{1}\right) E_{g}\right],
\end{aligned}
$$

$$
\begin{aligned}
\left(\widetilde{H}_{0}\right)_{r g}= & \frac{1}{2} E_{r}^{\dagger}\left(\nu n-\delta_{1}\right) E_{g}-\frac{\left|\Omega_{2}\right|^{2}}{\sqrt{2} \Omega^{2}} E_{r}^{\dagger} D\left(i \eta_{2}\right)(\nu n-\Delta) \\
& \times D^{\dagger}\left(i \eta_{2}\right) E_{g}-\frac{\left|\Omega_{1}\right|^{2}}{\sqrt{2} \Omega^{2}} E_{r}^{\dagger} D\left(i \eta_{1}\right) \nu n D^{\dagger}\left(i \eta_{1}\right) E_{g},
\end{aligned}
$$

where $\eta_{12}=\eta_{1}-\eta_{2}, \Delta=\delta_{1}-\delta_{2}$, and $n=a^{\dagger} a$ are used for notational simplicity. Examination of these equations gives us the conditions on $\theta_{j}$. In order to linearize the exponential dependences on vibrational phonon operators, we consequently choose $\theta_{j}=\zeta_{j}\left(a+a^{\dagger}\right)$ with $\zeta_{j}$ being real numbers under the constraints of $\zeta_{g}=\zeta_{r}$ and $\zeta_{e}=\zeta_{g}-\eta_{1}$. Hence, the exponential phase operators become displacement operators $E_{j}=D\left(i \zeta_{j}\right)$ which yield $\widetilde{H}_{0}$ given by

$$
\begin{gathered}
\left(\widetilde{H}_{0}\right)_{e r}=-i \nu \eta_{12} \frac{\Omega_{1}^{*} \Omega_{2}^{*}}{\sqrt{2} \Omega^{2}}\left(a^{\dagger}-a\right)+c_{e r}, \\
\left(\widetilde{H}_{0}\right)_{r g}=i \frac{\nu}{2}\left(\eta_{1}-\eta_{12} \frac{\left|\Omega_{2}\right|^{2}}{\Omega^{2}}\right)\left(a^{\dagger}-a\right)+c_{r g}, \\
\left(\widetilde{H}_{0}\right)_{e e}=\nu n+i \nu\left(\zeta_{e}+\frac{\eta_{12}\left|\Omega_{1}\right|^{2}}{\Omega^{2}}\right)\left(a^{\dagger}-a\right)+c_{e e}, \\
\left(\widetilde{H}_{0}\right)_{r r}=\nu n+i \frac{\nu}{2}\left(\zeta_{e}+\eta_{1}+\eta_{12} \frac{\left|\Omega_{2}\right|^{2}}{|\Omega|^{2}}\right)\left(a^{\dagger}-a\right)+c_{r r}, \\
\left(\widetilde{H}_{0}\right)_{g g}=\left(\widetilde{H}_{0}\right)_{r r}, \quad\left(\widetilde{H}_{0}\right)_{e g}=-\left(\widetilde{H}_{0}\right)_{e r} .
\end{gathered}
$$

Scalar constants $c_{i j}$ are given in Appendix A. Since $R_{e e}$ $+R_{r r}+R_{g g}=1$, first terms of each diagonal element add up to a contribution $\nu n$ in the transformed total Hamiltonian. One can also seek a similar decoupling of the internal and external degrees of freedom in the next (second) terms. It can be observed that if the coefficient of the momentum operator $i\left(a^{\dagger}-a\right)$ in $\left(\widetilde{H}_{0}\right)_{e e}$ would be the same as the corresponding one in $\left(\widetilde{H}_{0}\right)_{r r}$, the diagonal operators $|j\rangle\langle j|$ would be similarly uncoupled from $i\left(a^{\dagger}-a\right)$. Thus, we could now specify $\zeta_{e}$ to eliminate such terms completely. The requirement of the same momentum operator coefficients in the diagonal elements of $\widetilde{H}_{0}$ is satisfied if

$$
\frac{k_{1}}{k_{2}}=\frac{\left|\Omega_{2}\right|^{2}-2\left|\Omega_{1}\right|^{2}}{2\left|\Omega_{2}\right|^{2}-\left|\Omega_{1}\right|^{2}},
$$

under which choosing $\zeta_{e}=-\eta_{12}\left|\Omega_{1}\right|^{2} /|\Omega|^{2}$ cancels c.m. momentum terms. If the laser beam parameters are adjusted to satisfy this condition, the transformed Hamiltonian would be the same as the Hamiltonian of a single mode bosonic field interacting with a three-level system. Further specification of the laser parameters would allow us to engineer various interaction schemes. In this paper, we shall examine a case of counterpropagating beams $k_{1}=-k_{2}>0$, which gives 
$\left|\Omega_{1}\right|=\left|\Omega_{2}\right|$. We immediately find $\zeta_{g}=\zeta_{r}=0$ and $\zeta_{e}=\eta_{2}=$ $-\eta_{1} \equiv-\eta$. Transformed Hamiltonian of the system in this case becomes

$$
\begin{aligned}
\widetilde{H}= & \nu a^{\dagger} a+\sum_{j} \epsilon_{j} R_{j j}+\frac{1}{\sqrt{2}}\left[\frac{\Delta}{2}-i \nu \eta\left(a^{\dagger}-a\right)\right]\left(R_{e r}+R_{r e}\right. \\
& \left.-R_{e g}-R_{g e}\right)+\frac{1}{2}\left(-\nu \eta^{2}-\delta_{1}+\frac{\Delta}{2}\right)\left(R_{g r}+R_{r g}\right) .
\end{aligned}
$$

We introduced effective free energies of the ion as

$$
\begin{gathered}
\epsilon_{e}=\nu \eta^{2}-\frac{\Delta}{2}, \\
\epsilon_{r}=\frac{\nu \eta^{2}}{2}-\frac{\delta_{1}}{2}-\frac{\Delta}{4}+\frac{\Omega}{2}, \\
\epsilon_{g}=\frac{\nu \eta^{2}}{2}-\frac{\delta_{1}}{2}-\frac{\Delta}{4}-\frac{\Omega}{2} .
\end{gathered}
$$

Before going further let us consider some trivial cases of large detunings. If $\Delta$ is large enough, it can be seen immediately that the c.m. motion is decoupled from internal level dynamics. This may be used in generating particular states of c.m. motion by applying a large detuning $\Delta$ as a sudden perturbation after a demanded state is created. When $\delta_{1}$ is large but $\Delta=0$, lower levels of the ion $(g, r)$ become coupled through a classical field interaction while they interact with the upper level $(e)$ via vibrational phonon transitions. It can be shown that either one of the couplings, $(e-r$ or $e-g$ ), can be discarded under RWA and the system behaves effectively as a $V$-type three-level system. Below, we focus on a nontrivial quantum case of a weakly detuned system in which $\Delta=0$ and $\delta_{1}=-\nu \eta^{2} . \Delta=0$ can be true when lower levels of the ion are degenerate as we consider $\left|k_{1}\right|$ $=\left|k_{2}\right|$. However, there is no loss of generality here. More general case of nondegenerate levels can be treated by simply considering $k_{1}$ and $k_{2}$ to be proportional and rescaling the interaction coefficients in the Hamiltonian accordingly. In the weakly detuned case, classical field induced lower-level transitions cannot contribute to the transformed Hamiltonian. There remains four processes (other four are conjugate processes) in the form $a^{\dagger} R_{i j}$. In the interaction picture, they acquire phase factors of $\exp \left[-i t\left(\nu+\epsilon_{i}-\epsilon_{j}\right)\right]$. We have $\epsilon_{e}$ $-\epsilon_{r}=\epsilon_{e}-\epsilon_{g}=-\Omega / 2$. Taking $\Omega \sim 2 \nu$ (weak excitation regime), only processes of the ladder transitions would dominate the dynamical behavior of the system under the regimes where RWA is valid. The regime of validity here is $\nu$ $>\nu \eta / \sqrt{2}$, or $\eta<\sqrt{2}$. The RWA applied here works perfectly in the Lamb-Dicke regime $(\eta \ll 1)$ and can also be used beyond Lamb-Dicke limit (given by $\eta<1$ ). Determination of a certain value of Lamb-Dicke parameter below which RWA is perfectly valid requires comparison of predictions of RWA with those of the exact solution. Unitary transformation method developed above has an advantage that one has to deal with a less involved matrix representation of the Hamiltonian. The original trapped ion model contains the displace-

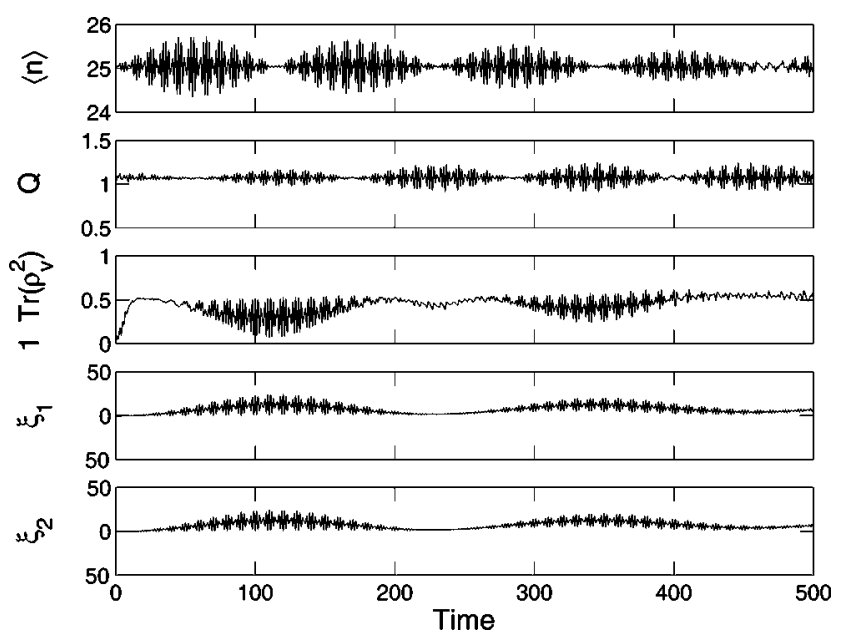

FIG. 2. Dynamical properties of vibrational phonons when $\eta$ $=0.1$ are determined by exactly solving the model via NDA for the initial condition $\psi(0)=|\alpha=5 i\rangle(|g\rangle-|r\rangle) / \sqrt{2}$. Time is dimensionless, scaled by $\sqrt{2} \nu \eta|\alpha|$, and $\theta=0$ is used.

ment operators with many nonzero elements in the Fock basis. The transformed cascade model on the other hand becomes a band matrix and allows for an efficient diagonalization procedure. We described such a numerical approach for the exact solution of a two-level system in Ref. [25]. Generalization of this numerical diagonalization algorithm (NDA) to the present situation is straightforward and summarized in Appendix C. We have performed extensive numerical study on RWA and NDA predictions. One comparison is exemplified in Figs. 2 and 3. Dynamical variables that are used in characterizing temporal properties of the threelevel ion are defined as follows. $\langle n\rangle$ is the mean number of phonons, $I=1-\operatorname{Tr}\left(\rho_{v}^{2}\right)$ is the impurity parameter [29], $Q$ $=\left(\left\langle n^{2}\right\rangle-\langle n\rangle^{2}\right) /\langle n\rangle$ is the Mandel's $Q$-parameter [30], and variances of quadratures are $\Delta Y_{i}^{2}$ where

$$
Y_{1}(\theta)=\frac{\left(a e^{-i \theta}+a^{\dagger} e^{i \theta}\right)}{\sqrt{2}}, \quad Y_{2}(\theta)=\frac{\left(a e^{-i \theta}-a^{\dagger} e^{i \theta}\right)}{\sqrt{2 i}} .
$$

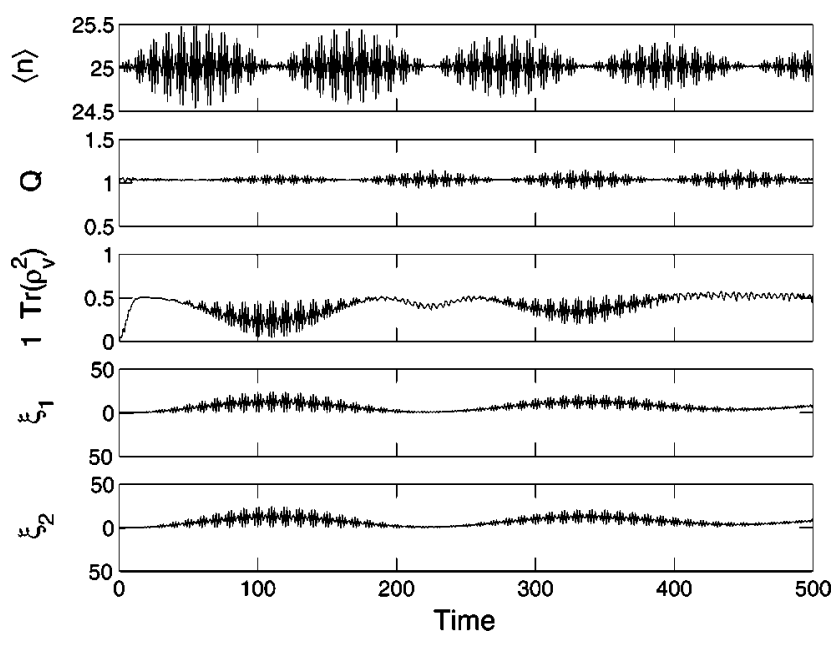

FIG. 3. Dynamical properties of vibrational phonons when $\eta$ $=0.1$ are determined under RWA for the initial condition $\psi(0)$ $=|\alpha=5 i\rangle(|g\rangle-|r\rangle) / \sqrt{2}$. Time is dimensionless, scaled by $\sqrt{2} \nu \eta|\alpha|$, and $\theta=0$ is used. 
When either of the parameters $\xi_{i}=\Delta Y_{i}^{2}-1 / 4$ becomes negative, the system is said to be in a squeezed state. In addition, we also consider level populations $\left\langle R_{j j}\right\rangle$ as well as $Q\left(\alpha, \alpha^{*}\right)=\left\langle\alpha\left|\rho_{v}\right| \alpha\right\rangle$, which is a quasiprobability function known as Husimi- $Q$ function [31]. Reduced density matrix $\rho_{v}$ of vibrational phonons is found by taking the trace of the density matrix of the total system over internal electronic levels. In Figs. 2 and 3, we see excellent agreement between RWA and NDA predictions. Deviations start to become more and more significant as $\eta$ increases. By examining a number of initial conditions and $\eta$, we reached a general conclusion that up to a critical Lamb-Dicke parameter $\eta_{c} \sim 1 /|\alpha|$, RWA can be used with very good agreement with the NDA. Here $\alpha$ is the initial coherent-state amplitude of the vibrational phonons. The range of perfect agreement is close but smaller than $\eta_{c}$. For $\alpha=2 i, 3 i, 5,10$, excellent quantitative agreement is found up to $\eta=0.3,0.2,0.07,0.08$ respectively. The phase of $\alpha$ plays a role in the optimum range since a wider range of validity is obtained for purely imaginary $\alpha$. For example, when 25 phonons are in a coherent state, $\eta_{c}=0.1$ and 0.2 , respectively, for real and imaginary $\alpha$. Same conclusions were reached for the $Q$ function, which can be expected as the $Q$ function behavior is closely related to the impurity parameter. For $\alpha=5$, we did not find any significant difference in $Q$ functions obtained by NDA and RWA up to $\eta=0.06$, beyond which small differences in heights of the peaks of the $Q$ function were observed. After $\eta=0.08$, quantitative differences became more appreciable but we still observed qualitatively the same behavior. We would prefer to know the impurity parameter as exactly as possible in order to identify the formation of macroscopic quantum superpositions. Therefore, we shall use RWA only in regimes where it is exact. In the vicinity of $\eta_{c}$ where quantitative deviations start to become significant despite the good qualitative agreement, we use NDA. It may also be mentioned that the standard approximations in LDL consist of the removal of the displacement operators via $D(i \eta) \sim 1$ or $D(i \eta) \sim 1+i \eta(a$ $\left.+a^{\dagger}\right)$. These are possible only if $2 \eta|\alpha| \ll 1$. Unitary transformation method under RWA extends this limit to $\eta_{c}|\alpha|$ $\sim 1$. Furthermore, with NDA, it can deal with the whole range of $\eta$. NDA may loose its practicality for small $\eta_{c}$ because for large $\alpha$, we would need to diagonalize large matrices. In those regimes, however, RWA works perfectly. Hence, it is good to have analytical solutions in such cases.

Finally, we present the Hamiltonian describing the underlying $\Xi$ configuration for the dynamics of the vibrational phonons while optically, the system is in $\Lambda$ configuration,

$$
\widetilde{H}=\nu n+\nu \eta^{2}+\nu\left(R_{r r}-R_{g g}\right)+V_{R W A},
$$

where

$$
V_{R W A}=-i \mu\left(a^{\dagger} R_{e r}-a^{\dagger} R_{g e}+\text { H.c. }\right)
$$

with $\mu=\nu \eta / \sqrt{2}$. The upper level of the ion (e) now becomes the intermediate level of the cascade as shown in Fig. 4. In this cascade model, all levels are linked resonantly with the same transition strengths.
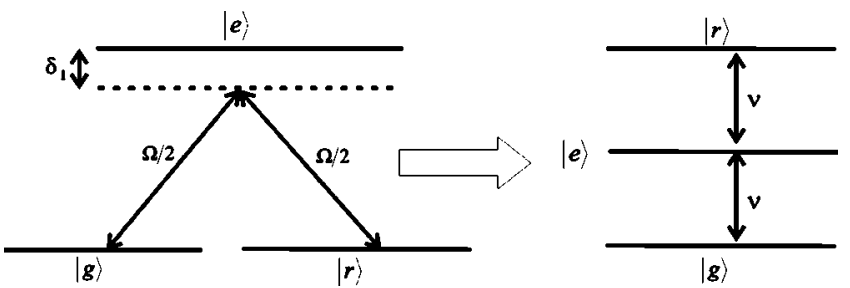

FIG. 4. Three-level ion interacting with two laser pulses in a $\Lambda$ configuration can be made unitarily equivalent to resonant cascade transitions of vibrational phonons for the appropriately chosen laser parameters as explained in the text.

A given state of vibrational phonons and the ion evolves in the $\Xi$ configuration according to the propagator in the interaction picture,

$$
K(t)=\left(\begin{array}{ccc}
\cos \Lambda t & -\mu S a^{\dagger} & -\mu S a \\
\mu a S & 1+\mu^{2} a C a^{\dagger} & \mu^{2} a C a \\
\mu a^{\dagger} S & \mu^{2} a^{\dagger} C a^{\dagger} & 1+\mu^{2} a^{\dagger} C a
\end{array}\right) .
$$

Here the basis is $\langle e| \rightarrow(1,0,0),\langle r| \rightarrow(0,1,0),\langle g| \rightarrow(0,0,1)$. For notational simplicity, we used $\Lambda=\mu \sqrt{2 a^{\dagger} a+1}$ and

$$
C=\frac{\cos \Lambda t-1}{\Lambda^{2}}, \quad S=\frac{\sin \Lambda t}{\Lambda} .
$$

Propagation of an initial state $\psi(0)$ of the original system can be expressed as

$$
\psi(t)=U_{0}^{\dagger} U_{E} e^{-i t\left(\tilde{H}-V_{R W A}\right)} K(t) U_{E}^{\dagger} \psi(0) .
$$

The two rightmost factors, $U_{E}(0)^{\dagger} \psi(0)=\widetilde{\psi}(0)$, act as the initial state for the cascade system, evolving by $K(t)$ into $\widetilde{\psi}(t)$, a state vector of the cascade. The exponential term and the prefactor $U_{0}$ stand for the interaction picture and the rotating frame back transformations, respectively. The timeindependent factor $U_{E}$ is the back transformation from the cascade to the original $(\Lambda)$ system. It redistributes the level $(e, r, g)$ amplitudes of the evolved state of the cascade and brings back multiple phonon transition effects through the shift of the amplitudes in momentum space. This can be seen clearly in the explicit exact solution below. We assume an initial state as a product state of the subsystems which is given to be

$$
\psi(0)=(x|g\rangle+y|r\rangle+z|e\rangle) \sum_{n=0}^{\infty} F_{n}|n\rangle .
$$

Here $F_{n}$ are the amplitudes of the Fock number states of vibrational phonons. This state is transformed to an initial state of the cascade so that

$$
\widetilde{\psi}(0)=\sum_{n=0}^{\infty}\left(A_{n}|e, n\rangle+B_{n}|r, n\rangle+C_{n}|g, n\rangle\right),
$$

where 


$$
\begin{gathered}
A_{n}=\frac{1}{\sqrt{2}} \sum_{m=0}^{\infty}\left[x F_{m} D_{n m}(i \eta)-y F_{m} D_{n m}(-i \eta)\right], \\
B_{n}=\frac{z}{\sqrt{2}} F_{n}+\frac{1}{2} \sum_{m=0}^{\infty}\left[x F_{m} D_{n m}(i \eta)+y F_{m} D_{n m}(-i \eta)\right], \\
C_{n}=\frac{z}{\sqrt{2}} F_{n}-\frac{1}{2} \sum_{m=0}^{\infty}\left[x F_{m} D_{n m}(i \eta)+y F_{m} D_{n m}(-i \eta)\right] .
\end{gathered}
$$

Matrix elements of the displacement operator $\langle m|D(\alpha)| n\rangle$ $=D_{m n}(\alpha)$ are the same as Franck-Condon factors for $\alpha$ $=i \eta$ and are given in terms of the Laguerre polynomials [31],

$$
D_{m n}(\alpha)=\sqrt{\frac{n !}{m !}} e^{-|\alpha|^{2} / 2} \alpha^{m-n} \mathcal{L}_{n}^{m-n}\left(|\alpha|^{2}\right) .
$$

It may be noted that for $F_{n}$ corresponding to an initial coherent state $\widetilde{\psi}(0)$ becomes a superposition of the states of the form $|\beta\rangle|a\rangle$ where $\beta=\alpha, \alpha \pm i \eta, a=e, r, g$. Denoting the amplitudes of the evolved transformed state $\widetilde{\psi}(t)$ by $A_{n}(t), B_{n}(t), C_{n}(t)$ in the Schrödinger picture, we find that the actual state vector of the system is given by

$$
\psi(t)=\sum_{n=0}^{\infty}\left(u_{n}|e, n\rangle+v_{n}|r, n\rangle+w_{n}|g, n\rangle\right),
$$

where

$$
\begin{gathered}
u_{n}=\frac{e^{-i \omega_{1} t}}{\sqrt{2}}\left[B_{n}(t)+C_{n}(t)\right], \\
v_{n}=\sum_{m=0}^{\infty} D_{m n}(i \eta)\left[-\frac{1}{\sqrt{2}} A_{m}(t)+\frac{B_{m}(t)-C_{m}(t)}{2}\right], \\
w_{n}=\sum_{m=0}^{\infty} D_{m n}(-i \eta)\left[\frac{1}{\sqrt{2}} A_{m}(t)+\frac{B_{m}(t)-C_{m}(t)}{2}\right] .
\end{gathered}
$$

The amplitudes $A_{n}(t), B_{n}(t), C_{n}(t)$ are given in Appendix B. This result shows explicitly the inter-relation of state vector amplitudes of the $\Lambda$ and $\Xi$ models. We can describe time dependence of the state vector of the $\Lambda$ system by examining the dynamics of the state vector of the cascade model. Below we shall use a quasiprobability distribution to discuss wave function temporal properties. It can be noted that for a negligibly small Lamb-Dicke parameter, only those amplitudes associated with the same number of vibrational phonons are inter-related, without any interference from amplitudes of different number of phonons. Time dependence of operator functions $\Theta$ can be similarly studied within the cascade model provided that the function is transformed accordingly. As we have $\langle\Theta\rangle(t)=\langle\psi(t)|\Theta| \psi(t)\rangle$ where

$$
\langle\Theta\rangle(t)=\left\langle\widetilde{\psi}(t)\left|e^{i t\left(\widetilde{H}-V_{R W A}\right)} U_{E}^{\dagger} \Theta U_{E} e^{-i t\left(\widetilde{H}-V_{R W A}\right)}\right| \widetilde{\psi}(t)\right\rangle,
$$

it is possible to describe dynamical behaviors of the operators of the trapped ion system using those of the cascade model as building blocks. Time dependences of the operators of the cascade model are well known and much simpler than the trapped ion system which involves multiple phonon transitions. For example,

$$
\begin{gathered}
U_{E}^{\dagger} R_{e e} U_{E}=\frac{1}{2}\left(1-R_{e e}\right)+\frac{1}{2} S_{r g}^{x}, \\
U_{E}^{\dagger} R_{g g} U_{E}=\frac{1}{4}\left(1+R_{e e}\right)-\frac{1}{2}\left(S_{r g}^{x}+\sqrt{2} S_{e g}^{x}-\sqrt{2} S_{e r}^{x}\right), \\
U_{E}^{\dagger} a^{\dagger} a U_{E}=a^{\dagger} a+\frac{\eta^{2}}{2}\left(1+R_{e e}-2 S_{r g}^{x}\right) \\
-\frac{\eta}{\sqrt{2}} i\left(a^{\dagger}-a\right)\left(S_{r e}^{x}-S_{e g}^{x}\right) .
\end{gathered}
$$

Here the dipole (spin) operators are introduced by $S_{i j}^{x}=\left(R_{i j}\right.$ $\left.+R_{j i}\right) / 2$. Time-varying properties of vibrational phonons and level populations are affected by the propagation of such dipole operators under ladder transitions. In particular, the excited state time evolution is directly influenced by the dynamics of $S_{r g}^{x}$. Lower-level dynamics carry the combined effect of all three dipoles. Looking at the cascade dynamics of those dipoles, we can easily understand the underlying time scales and simpler behaviors of the resultant, complex dynamics of level populations. There appears to be contributions from both the dipole and the momentum terms in the mean number of phonons. It is illuminating to compare such terms with those of the transformed Hamiltonian. Just before the rotating wave approximation made, we have the relation

$$
\begin{aligned}
U_{E}^{\dagger} a^{\dagger} a U_{E}= & \frac{U_{E}^{\dagger} H U_{E}}{\nu}+\left(1-\frac{\eta^{2}}{2}\right) R_{g g} \\
& -\left(1+\frac{\eta^{2}}{2}\right) R_{r r}-\eta^{2} S_{r g}^{x} .
\end{aligned}
$$

The first term can be split into two. One part is the Hamiltonian obtained after RWA, and the other part consists of the remaining counterrotating terms. The RWA part is a constant for the cascade wave function and does not contribute to the dynamics. Considering small $\eta$, the major dynamical contributions come from the counterrotating terms and the inversion $R_{r r}-R_{g g}$ terms. In cases where these are negligible, other terms $\left(R_{g g}+R_{r r}\right.$ and the last term) can also influence the dynamics in the order of $\eta^{2}$.

\section{RESULTS AND DISCUSSIONS}

We consider an initial state of the system in which the vibrational phonon subsystem is in a coherent state and the ion is in a superposition state of its lower levels so that

$$
\psi(0)=\frac{1}{\sqrt{2}}(|g\rangle-|r\rangle) \sum_{n=0}^{\infty} F_{n}(\alpha)|n\rangle,
$$




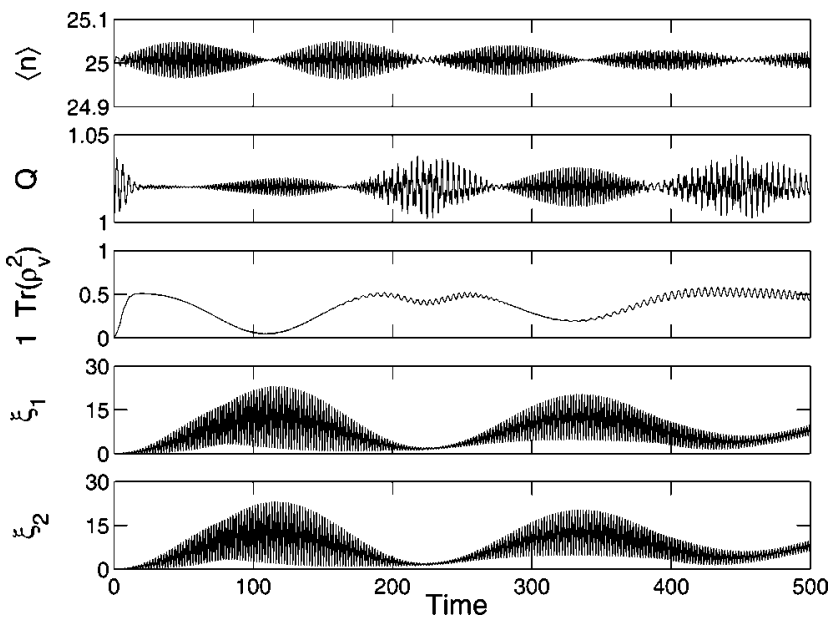

FIG. 5. Dynamical properties of vibrational phonons for the initial condition $\psi(0)=|\alpha=5\rangle(|g\rangle-|r\rangle) / \sqrt{2}$ in the Lamb-Dicke limit with $\eta=0.01$. Time is dimensionless, scaled by $\sqrt{2} \nu \eta|\alpha|$, and $\theta=0$ is used.

where

$$
F_{n}(\alpha)=e^{-|\alpha|^{2} / 2} \frac{\alpha^{n}}{\sqrt{n !}} .
$$

The state in Eq. (23) transforms into an initial state for the cascade model in which the ion can be considered approximately in the intermediate state $|e\rangle$ of the cascade for $|\alpha|$ $\gg \eta$. It is known that a three-level cascade model, prepared initially in its middle level, produces a two-component cat state [32]. That is why we consider that particular initial preparation of the original $\Lambda$ system.

For $\alpha=5$, and $\eta=0.01$, time dependence of the original $\Lambda$ system is shown in Figs. 5 and 6. In all figures, time is scaled by $\sqrt{2} \nu \eta|\alpha|$ and is dimensionless. For the initial condition where the cascade is prepared in its intermediate state $|e\rangle$, time variances of spin operators in the cascade model are shown in Fig. 7. Note that the regular collapse and double revival patterns of the level populations in a cascade
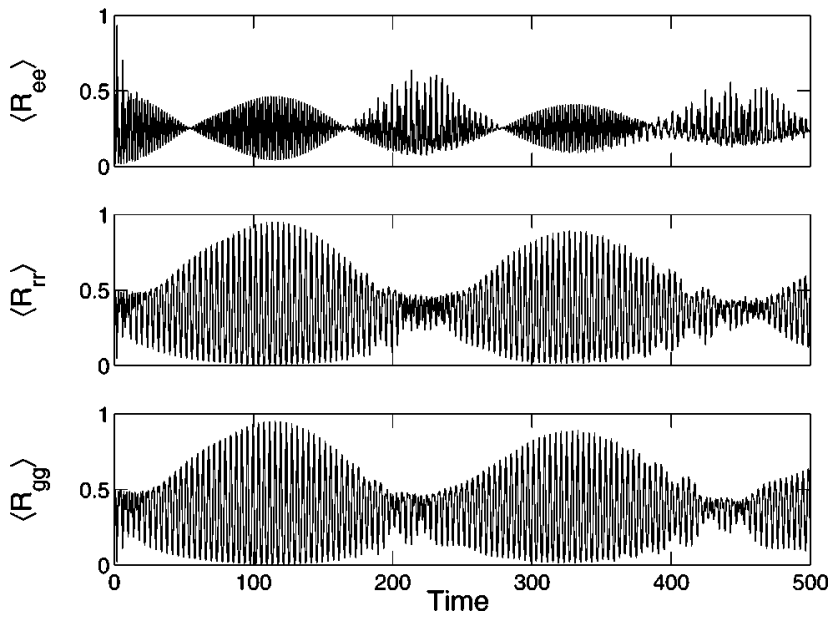

FIG. 6. The same as in Fig. 5 but for the internal level populations of the ion.

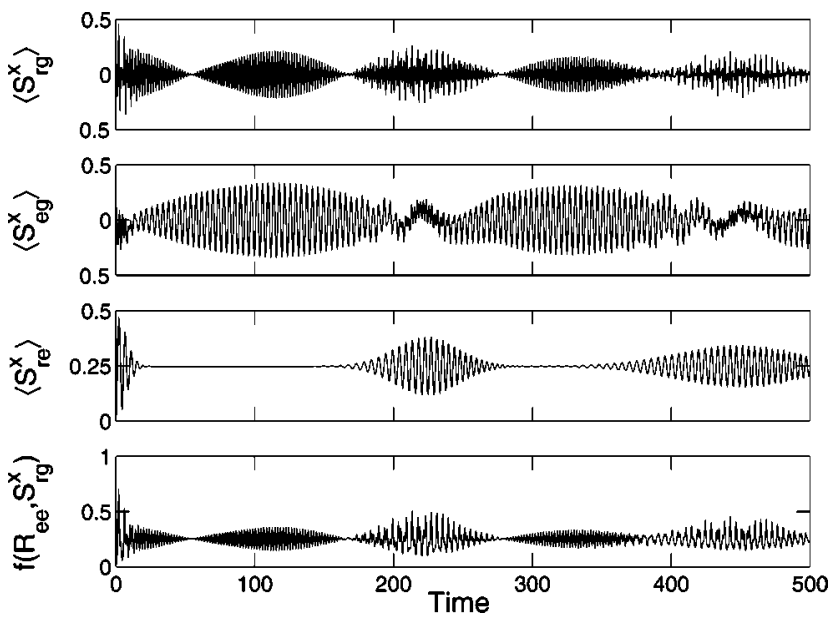

FIG. 7. Time evolution of spin operators of the cascade model prepared initially in state $U_{E}^{\dagger} \psi(0)$ with $\psi(0)=|\alpha=5\rangle(|g\rangle-|r\rangle) / 2$ and $\eta=0.01$. The bottom frame shows a combined evolution of $R_{e e}$ with $S_{r g}^{x}$ such that $f\left(R_{e e}, S_{r g}^{x}\right)=\left\langle\left(1-R_{e e}+S_{r g}^{x}\right) / 2\right\rangle$. Time is dimensionless, scaled by $\sqrt{2} \nu \eta|\alpha|$.

model are well known and not shown here (see, for example, Ref. [32]). When only the middle level is populated initially, the mean number of phonons cannot change in a cascade system where the levels are linked resonantly at equal strengths. The small and rapidly reviving oscillations we observe in $\langle n\rangle$ are due to the counterrotating processes as we argued for Eq. (22). The other terms in Eq. (22) cannot make significant contribution. The small Lamb-Dicke parameter used here diminishes any dynamical influences of the level populations and the dipole term $S_{r g}^{x}$ in $\langle n\rangle$. Inversion also cannot contribute as it is vanishing for the cascade model. Hence, we directly observe the dynamics of counterrotating terms of the $H$ in the $\langle n\rangle$ evolution. The Mandel $Q$ parameter indicates the super-Poissonian character of the system and the quadratures show no squeezing, also similar with the cascade model.

Behavior of level populations can be more directly understood in terms of the cascade model. Upper-level dynamics of the $\Lambda$ system is characterized by the $S_{r g}^{x}$ evolving under the $\Xi$ model as can be seen clearly by comparing Figs. 6 and 7. The bottom frame in Fig. 7 shows the combined evolution of $R_{e e}$ and $S_{r g}^{x}$ of the cascade model. The result is same with the $\left\langle R_{e e}\right\rangle$ in the $\Lambda$ configuration as expected. Similarly, we understand that the broad oscillatory nature of $S_{e g}^{x}$ under the cascade model shapes the lower level dynamics of the $\Lambda$ system.

The behavior of the impurity parameter of the $\Lambda$ system is similar to that of the cascade model (see, e.g., Ref. [32]) prepared initially in its intermediate state. Around $t=100$, where impurity is almost zero, the phonon subsystem becomes disentangled from the internal degrees of freedom. This pure state can be examined by the Husimi- $Q$ function as shown at several times in Fig. 8.

Initially, $Q$ function is a single-peak Gaussian distribution centered at the $\alpha=5$. As time progresses, it splits into three distinct peaks, rotating counterclockwise on a circle of radius $\sim|\alpha|$ with different speeds. Only contour lines correspond- 

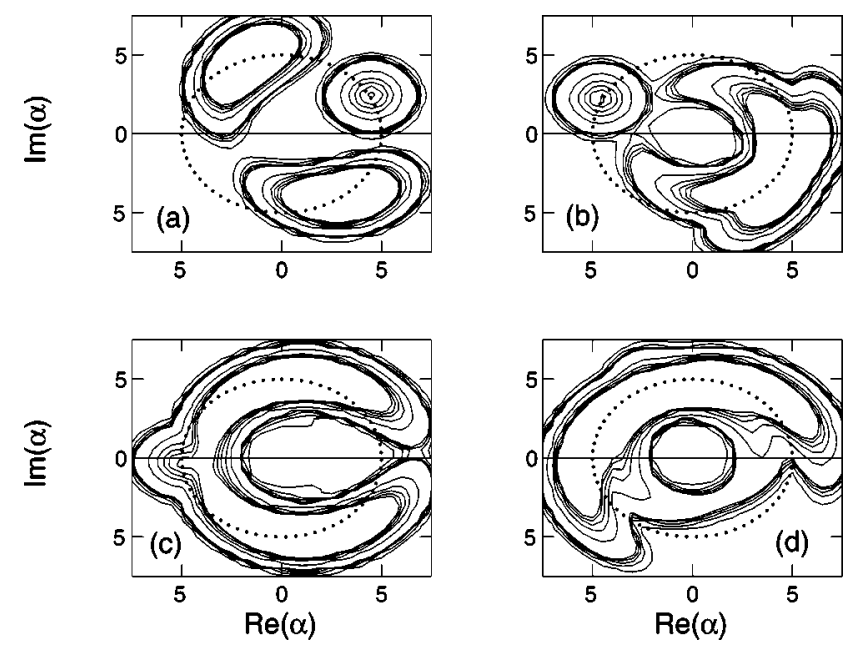

FIG. 8. Contour lines of the vibrational phonon $Q$ function in the complex $\alpha$ plane at dimensionless times (a) $t=100$, (b) $t$ $=225$, (c) $t=325$, and (d) $t=400$. Contours corresponding to heights beyond 0.009 and between 0.00009 and 0.001 are not shown for clarity in the figure. The initial condition and the parameters are the same as in Fig. 5.

ing to very low heights are shown in Fig. 8 as the peak (component) with circular contours is negligibly small compared to the other two. At time $t=100$ there is no interference among the components and the pure state is, in principle, a three-component Schrödinger cat. On the other hand, as one peak is negligible, the state is approximately a twocomponent cat state. At $t=225$, two components collide, but the small peak survives. Even though there is no cat state now, the impurity parameter in Fig. 5 makes a local minimum. A similar situation happens at $t=325$ where the impurity has another and deeper local minimum. From Fig. 8, we see that the small peak collides with the other two at this time while the other two are at their extreme phase space separation from each other. After that, all three collide as given at $t=400$. It should be mentioned that collisions never occur at the starting location due to the presence of the moving small peak. The negligible component affects the locations of the other two. The field quasiprobability cannot regain its original value at all. Therefore, we observe neither normal nor superrevivals of $\langle n\rangle$ in this case.

Furthermore, different from cavity QED models, there is no obvious relation between the splitting-combination times of the components of the $Q$ function and the revival times of $\langle n\rangle$. In particular, the occurrence of cat state at $t=100$ does not coincide with the half-revival time of the mean number of phonons. It does, however, coincide with the half-revival time of $\langle n\rangle$ of the cascade model. This dynamical relation between the $Q$ function and $\langle n\rangle$ is hidden in the trapped ion system due to the contributions of multiple vibrational phonon transitions, yet it can be revealed by examining the underlying cascade transitions.

The differences between the $Q$ functions of cavity QED cascade model and the trapped ion in $\Lambda$ configuration reflect the linear superposition effect of the back unitary transform in the state vector amplitudes as we have argued before. For such a small $\eta=0.01$, ignoring the third peak, we just have a
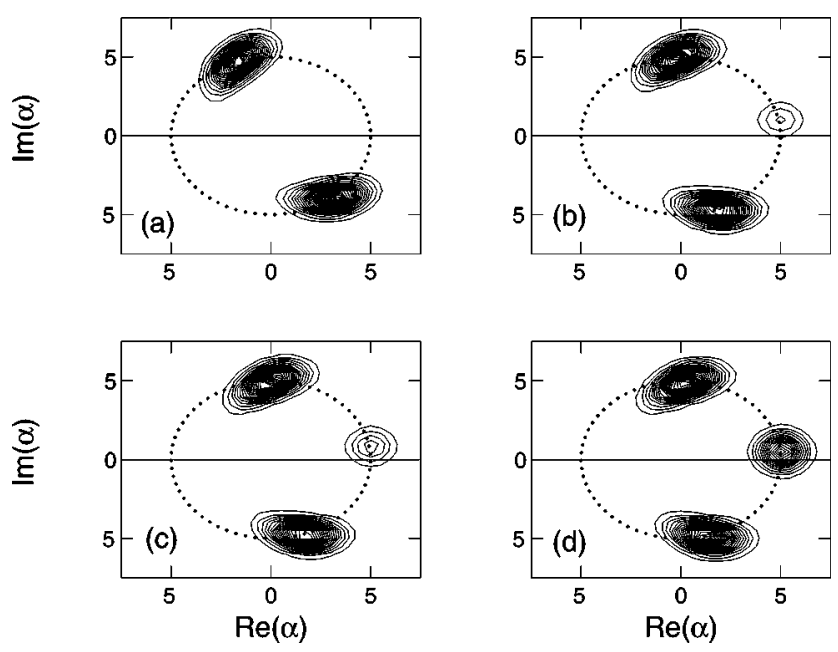

FIG. 9. Contour lines of the $Q$ function at dimensionless time $t=100$ for (a) $\eta=0.01$, (b) $\eta=0.025$, (c) $\eta=0.03$, and (d) $\eta$ $=0.05$.

two-component cat state similar to the cascade, with only difference in the rotational speeds and positions of the peaks. A more striking difference would be to emphasize the presence of the small peak as an additional third component to the cat state. We can achieve this objective by considering even slightly larger $\eta$. The sensitive dependence of peaks on Lamb-Dicke parameter should be expected since it introduces momentum space displacements in $U_{E}$, and also directly determines the interaction coefficient $\mu$ of the cascade model. We show the $\eta$ dependence of peaks in Fig. 9.

As $\eta$ increases, the height of the peak associated with circular contours increases. At $\eta=0.01$ the cat state has two components. The negligible third component cannot be noticed in the scale used in present figures. When $\eta=0.025$, small peak becomes visible and when $\eta=0.03$, it becomes a reasonably significant component. We could make it even higher than the other two peaks by continuing to increase $\eta$ at the cost of increasing the impurity. However, beyond $\eta$ $=0.05$, the impurity becomes too high to classify this state as a cat state anymore. Correlations of internal and external degrees of freedom of the ion do not become disentangled completely and the multiple-component superposition state becomes an incoherent superposition. The trap frequency or the laser frequency can be used to pick $\eta$ for the desired number of components in the cat state. We note that if the laser frequency is chosen as the control parameter, then in order to maintain the condition $\delta_{1}=-\nu \eta^{2}$, which is independent of $\nu$, detunings should also be adjusted accordingly. In Fig. 10, we present results obtained by NDA for a general initial condition of the form

$$
\psi(0)=\left(\cos \theta_{0}|g\rangle+e^{i \phi} \sin \theta_{0}|r\rangle\right)|\alpha\rangle .
$$

Here three comparable components are obtained both at small and at large $\eta$. We see that impurity parameter can be significantly lowered down to $\sim 0.1$. Such states can be considered as almost pure [11,32] and identified to be threecomponent cat states with comparable peaks. A similar structure of three components but with one of them as stationary 

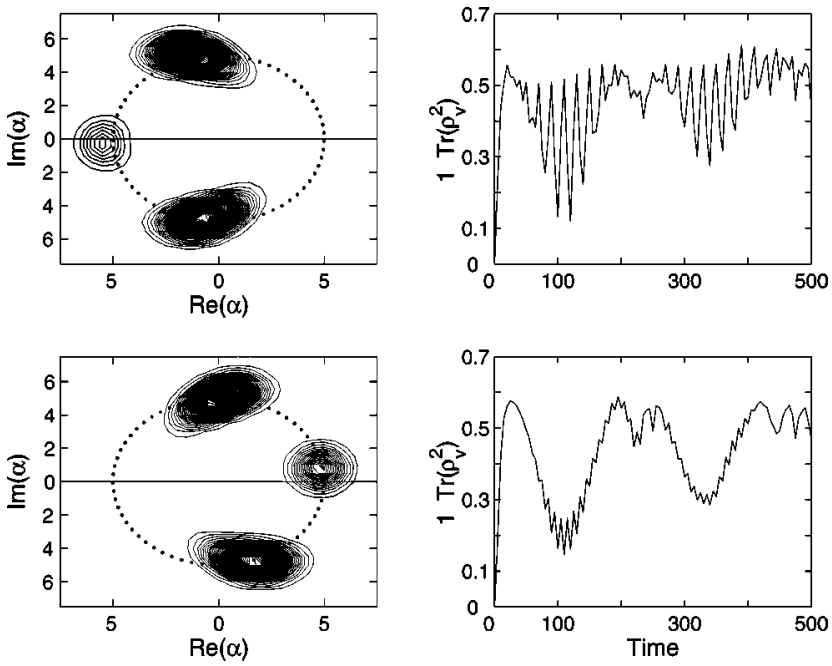

FIG. 10. $Q$ functions at $t=100$ and the time dependence of the impurity parameters are shown for two different initial conditions. In both figures $\alpha=5, \theta_{0}=\pi / 4$. Upper figures are for $\phi=\pi / 4, \eta$ $=0.1$ and the lower ones are for $\phi=\pi / 2, \eta=0.03$. All figures are obtained by the exact solution via NDA. Time is dimensionless, scaled by $\sqrt{2} \nu \eta|\alpha|$.

and not interfering with the others was found in cavity QED systems [32] and in two-level trapped ions in optical cavities [11]. The states in Ref. [32] are not identified to be cat states as they have much larger impurity parameters $(I \sim 0.3-0.5)$ and are not interfering. In our results, we observe that all three components are time dependent and interact with each other. In Ref. [32], it has been concluded that in order to reduce the impurity down to $I \sim 0.1$, one needs to eliminate the effect of the third level via reducing the dipole moment coupling or by using large detuning. This approach removes the stationary noninteracting component. In the present case, we show that it is possible to increase the purity when all three levels are contributing to the ion dynamics and all three components are present.

Let us also note that in our extensive numerical and analytical studies for a wide range of parameters, we have found that the number of components in the cat states as well as their amplitudes can be tuned by varying the magnitude and phase of $\alpha, \theta_{0}$, and $\phi$ in addition to $\eta$. As the effects are similar, we shall only mention that there are alternative routes leading to similar superpositions and effects which may provide flexibility in experiments.

\section{CONCLUSION}

We have examined the problem of trapped three-level ion interacting with two laser beams in $\Lambda$ configuration. A general family of unitary transformations is developed which permits analytical study of multiple vibrational phonon transitions in the actual problem via two-phonon transition models. Multiple transitions are contained within a transforming matrix of momentum space displacement operators. Various choices of system parameters in the transformed two-phonon Hamiltonian result in already known three-level interaction schemes of cavity QED. In addition to their configurations, interactions among internal levels can also be tailored to occur via quantum or classical channels again by choosing parameters of the system such as detunings, directions, and frequencies of the laser beams.

For a particular set of system parameters under the weak excitation and Lamb-Dicke regimes, a ladder-type twophonon transition model can be constructed. Summarizing the parameters, the driving beams are antiparallel and weakly red detuned from the upper level by the same amount if the lower levels are degenerate. It may be noted that LambDicke limit here is employed only to solve the problem analytically. Presence of counter rotating terms were treated numerically. Their inclusion did not destroy the cat state. It has been recently argued that when the excited level is eliminated adiabatically, two component cat states may still appear beyond the Lamb-Dicke limit [33]. Besides, the cat states are generated here in a single step in a time determined by the cascade model. Therefore, going beyond Lamb-Dicke limit to decrease preparation time does not seem to be as essential as it might be for other sequential schemes.

Complex temporal properties of the original system are now understood within the reduced cascade problem. Using the known results of $\Xi$ configuration, we have discussed time dependence of the trapped ion in $\Lambda$ configuration assuming that the vibrational phonons are given in a coherent state initially. In particular, time evolution of level populations and mean number of phonons are explained.

Furthermore, identifying the underlying cascade model, we predicted the existence of two- and three-component Schrödinger cat states. We have found that for the same initial preparation of the system, where the ion is given in a superposition of its lower levels, one can obtain either twoor three-component cat states depending on the Lamb-Dicke parameter. At larger Lamb-Dicke parameters, a third component appears and contributes more significantly with the increase of $\eta$. Trap frequency or laser frequency can be used to adjust $\eta$ as a knob for this effect. Cascade model characterization also permits us to determine when cat states appear. We have shown that cat states of the original $\Lambda$ system occur at half-revival time of the cascade model. It is argued that a large detuning of the lasers from the upper level as a sudden perturbation results in dynamical decoupling of the phonon subsystem from internal levels. This may be used to keep the cat states for detection and other applications once they are generated.

Unitary transformation method has been developed here for a trapped three-level ion optically driven in $\Lambda$ configuration. We have illustrated the method for the case of underlying cascade dynamics of vibrational phonons. This method can be easily adopted to three-level ions in other optical configurations (such as $\Xi, V$ ) or to engineer different underlying dynamical mechanisms for vibrational ions. Knowing simple unitary dynamical equivalents of trapped ion systems is desirable for understanding complex temporal behaviors, making predictions, and designing system parameters for quantum state generation, as well as extending standard approximate treatments. Unitary transformation method for the three-level ion eliminates the need for standard approximate schemes relying on adiabatic elimination and Lamb- 
Dicke regimes. We hope the presented method and the results can be useful for the ongoing theoretical and experimental efforts in trapped ion physics.

\section{ACKNOWLEDGMENT}

The author would like to thank Dr. L. You for helpful discussions.

\section{APPENDIX A: CONSTANTS OF $\widetilde{H}_{0}$}

The constants $c_{i j}$ in the general expression of the transformed free Hamiltonian $\widetilde{H}_{0}$ in Eq. (10) are given below:

$$
\begin{gathered}
c_{e r}=-\frac{\Omega_{1}^{*} \Omega_{2}^{*}}{\sqrt{2} \Omega^{2}}\left(2 \zeta_{1} \nu \eta_{12}+\nu \eta_{12}^{2}-\Delta\right), \\
c_{r g}=\zeta_{1} \nu\left(\eta_{1}-\eta_{12} \frac{\left|\Omega_{2}\right|^{2}}{\Omega^{2}}\right)-\frac{\delta_{1}}{2}+\frac{\Delta\left|\Omega_{2}\right|^{2}}{2 \Omega^{2}} \\
+\frac{\nu}{2}\left(\eta_{1}^{2}-\eta_{12}^{2} \frac{\left|\Omega_{2}\right|^{2}}{\Omega^{2}}\right), \\
c_{e e}=\nu \zeta_{e}^{2}+\frac{\left|\Omega_{1}\right|^{2}}{\Omega^{2}}\left(2 \zeta_{e} \nu \eta_{12}+\nu \eta_{12}^{2}-\Delta\right), \\
c_{r r}=\nu \zeta_{e}^{2}+\nu \zeta_{e} \eta_{1}+\frac{\nu \eta_{1}^{2}-\delta_{1}}{2}+\left(\nu \zeta_{e} \eta_{12}+\frac{\nu \eta_{12}^{2}-\Delta}{2}\right) \frac{\left.\Omega_{2}\right|^{2}}{\Omega^{2}} \\
c_{g g}=c_{r r}, \quad c_{e g}=-c_{e r} .
\end{gathered}
$$

\section{APPENDIX B: TIME DEPENDENCE OF WAVE FUNCTION AMPLITUDES IN CASCADE MODEL}

The time dependence of the wave function amplitudes $A_{n}(t), B_{n}(t), C_{n}(t)$ is given below in the Schrödinger picture. $A_{n}, B_{n}, C_{n}$ used without any explicit time dependence are those given at $t=0$ :

$$
\begin{aligned}
A_{n}(t)= & {\left[A_{n} \cos (\mu t \sqrt{2 n+1})-\left(B_{n-1} \sqrt{\frac{n}{2 n+1}}\right.\right.} \\
& \left.\left.+C_{n+1} \sqrt{\frac{n+1}{2 n+1}}\right) \sin (\mu t \sqrt{2 n+1})\right] e^{-i \nu t\left(n+\eta^{2}\right)} \\
B_{n}(t)= & \left\{A_{n+1} \sqrt{\frac{n+1}{2 n+3}} \sin (\mu t \sqrt{2 n+3})\right. \\
& +B_{n}\left[1-\frac{n+1}{2 n+3}[1-\cos (\mu t \sqrt{2 n+3})]\right] \\
& \left.-C_{n+2} \frac{\sqrt{(n+1)(n+2)}}{2 n+3}[1-\cos (\mu t \sqrt{2 n+3})]\right\} \\
& \times e^{-i \nu t\left(n+1+\eta^{2}\right)},
\end{aligned}
$$

$$
\begin{aligned}
C_{n}(t)= & \left\{A_{n-1} \sqrt{\frac{n}{2 n-1}} \sin (\mu t \sqrt{2 n-1})\right. \\
& +C_{n}\left[1-\frac{n+1}{2 n-1}[1-\cos (\mu t \sqrt{2 n-1})]\right] \\
& \left.-B_{n-2} \frac{\sqrt{n(n-1)}}{2 n-1}[1-\cos (\mu t \sqrt{2 n-1})]\right\} \\
& \times e^{-i \nu t\left(n-1+\eta^{2}\right)} .
\end{aligned}
$$

\section{APPENDIX C: NUMERICAL DIAGONALIZATION ALGORITHM}

Numerical diagonalization algorithm starts by preparation of the transformed initial state given by Eq. (19). Introducing $m$ as the truncation of the Fock space, we write the column vectors $A=\left(A_{1}, A_{2}, \ldots, A_{m}\right)^{T}$, etc., so that the initial transformed state can be expressed as $\widetilde{\psi}(0)=(A, B, C)^{T}$. In the same representation, the transformed Hamiltonian $\widetilde{H}$ in Eq. (12) becomes

$$
\widetilde{H}=\left(\begin{array}{lll}
H_{e e} & H_{e r} & H_{e g} \\
H_{r e} & H_{r r} & H_{r g} \\
H_{g e} & H_{g r} & H_{g g}
\end{array}\right)
$$

Here, $H_{a b}$ with $a, b=e, r, g$ are the $m \times m$ blocks of the $\widetilde{H}$; $H_{a a}=\epsilon_{a}$ are diagonal matrices corresponding to effective free energy part of the $\widetilde{H}$; and $H_{r g}=H_{g r}=0$ are zero matrices. The elements of the interaction matrices are simply proportional to the elements of the momentum operator $i\left(a^{\dagger}\right.$ $-a)$ in the Fock number basis and thus $H_{e r}, H_{e g}, H_{r e}, H_{g e}$ are tri-diagonal matrices. Therefore, the $\widetilde{H}$ is a band matrix with nonvanishing nine diagonals. We numerically find the eigenvalues and eigenvectors of $\widetilde{H}$. The eigenvectors are the columns of the diagonalizing matrix $V$. Writing the eigenvalue vector to be $W$, the propagating kernel is found to be $K(t)=V \exp (-i W t) V^{-1}$. Time evolved transformed state vector becomes $\widetilde{\psi}(t)=(A(t), B(t), C(t))^{T}=K(t) \widetilde{\psi}(0)$. Finally, we get the evolved actual state vector via $\psi(t)$ $=U_{E} \widetilde{\psi}(t)$ (up to an unimportant phase factor) as in Eq. (21). In the case of RWA, which yields the cascade transitions model, $H_{e r} \sim a^{\dagger}$ and $H_{e g} \sim a$. NDA is still applicable but the analytical results are also available. The propagating kernel is analytically determined and $A(t), B(t), C(t)$ are explicitly given in Appendix B. The truncation is chosen so that $m$ $\gg|\alpha \pm i \eta|^{2}$. We consider successively larger Fock spaces in order to check the convergence and the accuracy of the truncation. For larger $\alpha$, NDA demands more numerical resources. As long as we are in their regime of validity, as in the case of large $\alpha$ with small $\eta_{c}$, results of RWA allow for more efficient numerical treatment. 
[1] J.I. Cirac, A.S. Parkins, R. Blatt, and P. Zoller, Adv. At., Mol., Opt. Phys. 37, 237 (1996).

[2] D.J. Wineland, C.R. Monroe, C. Sackett, D. Kielpinski, M. Rowe, V. Meyer, and W. Itano, Ann. Phys. (N.Y.) 9, 851 (2000)

[3] D.J. Wineland, C.R. Monroe, W.M. Itano, D. Leibfried, B.E. King, and D.M. Meekhof, J. Res. Natl. Inst. Stand. Technol. 103, 259 (1998).

[4] C.A. Sackett, D. Kielpinski, B.E. King, C. Langer, V. Meyer, C.J. Myatt, M. Rowe, Q.A. Turchette, W.M. Itano, D.J. Wineland, and I.C. Monroe, Nature (London) 404, 256 (2000).

[5] D.M. Meekhof, C. Monroe, B.E. King, W.M. Itano, and D.J. Wineland, Phys. Rev. Lett. 76, 1796 (1996).

[6] A. Ben-Kish, B. DeMarco, V. Meyer, M. Rowe, J. Britton, W.M. Itano, B.M. Jelenkovic, C. Langer, D. Leibfried, T. Rosenband, and D.J. Wineland, Phys. Rev. Lett. 90, 037902 (2003).

[7] C. Monroe, D.M. Meekhof, B.E. King, and D.J. Wineland, Science 272, 1131 (1996).

[8] C. Roos, T. Zeiger, H. Rohde, H.C. Nagerl, J. Eschner, D. Leibfried, F. Schmidt-Kaler, and R. Blatt, Phys. Rev. Lett. 83, 4713 (1999).

[9] E. Schrödinger, Naturwissenschaften 23, 812 (1935). For an English translation, see Quantum Theory and Measurement, edited by J.A. Wheeler and W.H. Zurek (Princeton University Press, Princeton, NJ, 1983).

[10] H. Zeng, F. Lin, Y. Wang, and Y. Segawa, Phys. Rev. A 59, 4589 (1999).

[11] V. Bužek, G. Drobný, M.S. Kim, G. Adam, and P.L. Knight, Phys. Rev. A 56, 2352 (1997).

[12] A. Ben-Kish, B. DeMarco, V. Meyer, M. Rowe, J. Britton, W.M. Itano, B.M. Jelenković, C. Langer, D. Leibfried, T. Rosenband, and D.J. Wineland, e-print quant-ph/0208181.

[13] R.L. de Matos Filho and W. Vogel, Phys. Rev. Lett. 76, 608 (1996).
[14] J.C. Retamal and N. Zagury, Phys. Rev. A 55, 2387 (1997).

[15] O. Castaños, R. Jáuregui, R. López-Peña, J. Recamier, and V.I. Man'ko, Phys. Rev. A 55, 1208 (1997).

[16] J.F. Poyatos, J.I. Cirac, R. Blatt, and P. Zoller, Phys. Rev. A 54, 1532 (1996).

[17] C.C. Gerry, Phys. Rev. A 55, 2478 (1997).

[18] S.B. Zheng and G.C. Guo, Eur. Phys. J. D 1, 105 (1998).

[19] S.B. Zheng, Phys. Rev. A 58, 761 (1998).

[20] E.T. Jaynes and F.W. Cummings, Proc. IEEE 51, 89 (1963).

[21] H. Moya-Cessa, A. Vidiella-Barranco, J.A. Roversi, D.S. Freitas, and S.M. Dutra, Phys. Rev. A 59, 2518 (1999).

[22] I.Sh. Averbukh, Phys. Rev. A 46, R2205 (1992).

[23] Mao-Fa Fang, S. Swain, and P. Zhou, Phys. Rev. A 63, 013812 (2000); H. Moya-Cessa, A. Vidiella-Barranco, J.A. Roversi, and M.S. Dutra, J. Opt. B: Quantum Semiclassical Opt. 2, 21 (2000); D. Jonathan, M.B. Plenio, and P.L. Knight, Phys. Rev. A 62, 042307 (2000); H. Moya-Cessa, D. Jonathan, and P.L. Knight, quant-ph/0110167; X.X. Yang and Y. Wu, Chin. Phys. Lett. 19, 1625 (2002); L.M. Arevalo-Aguilar and H. MoyaCessa, Phys. Rev. A 65, 053413 (2002).

[24] P. Aniello, A. Porzio, and S. Solimeno, e-print quant-ph/0207151.

[25] Ö.E. Müstecaplioğlu and L. You, Phys. Rev. A 65, 033412 (2002).

[26] S.B. Zheng and G.C. Guo, Quantum Semiclassic. Opt. 9, L45 (1997).

[27] H. Moya-Cessa, S. Wallentowitz, and W. Vogel, Phys. Rev. A 59, 2920 (1999).

[28] L. Allen and J.H. Eberly, Optical Resonance and Two Level Atoms (Dover, New York, 1987).

[29] J. Gea-Banacloche, Phys. Rev. Lett. 65, 3385 (1990).

[30] L. Mandel, Opt. Lett. 4, 205 (1979).

[31] K.E. Cahill and R.J. Glauber, Phys. Rev. 177, 1857 (1969).

[32] P.L. Knight and B.W. Shore, Phys. Rev. A 48, 642 (1993).

[33] M. Feng, e-print quant-ph/0103112. 